\title{
LA PRODUCCIÓN MINERA DE LITIO EN AMÉRICA LATINA Y EL ASCENSO ECONÓMICO DE CHINA Y DE ASIA ORIENTAL
}

\author{
JULIÁN ZÍCARI \\ Universidad de Buenos Aires
}

Recepción manuscrito: 14 de enero de 2015

Aceptación versión final: 18 de marzo de 2015

\begin{abstract}
RESUMEN El trabajo aborda los vínculos de tenencia, producción y consumo del litio y los problemas que implica el neoextractivismo en Sudamérica a partir de la creciente demanda de China y de Asia Oriental. Para hacer esto se divide el trabajo en dos partes. En la primera, se señala cómo fue la dinámica del mercado del litio desde la década de 1990 hasta la actualidad, mientras que, en la segunda parte se trata de explicar los patrones de funcionamiento del mercado del litio entre regiones, abordando cómo es el actual vínculo entre Latinoamérica y Asia Oriental. Finalmente, se cierra el trabajo con algunas conclusiones al respecto.
\end{abstract}

palabras CLAVE Minería, litio, neoextractivismo, China, Latinoamérica.

ABSTRACT The paper deals with the links of ownership, production and consumption of lithium and the problems involved in neo-extractivism in South America from the growing demand in China and East Asia. To do this work, is divided into two parts. In the first, is pointed out how was the dynamic lithium market from the 90 s to the present, while in the second part is try to explain the patterns of market performance lithium across regions, addressing how is the current link between Latin America and East Asia. Finally, is ending this work some conclusions on the matter.

KEYWORDS Mining, lithium, neo-extractivism, China, Latin America.

CODIFICACIÓN JEL F13, O14, Q11, FO2.

\section{INTRODUCCIÓN}

El ascenso económico de Asia Oriental es una realidad innegable desde hace décadas y que se reforzó en los últimos años con el espectacular crecimiento de China. De allí que la demanda de recursos naturales por parte de Asia Oriental haya sido una de las características del proceso, provocando no sólo aumentos de precios, sino también una búsqueda desesperada de nuevas fuentes de abastecimiento para sostenerlo. En este caso, América Latina se ha vuelto un objetivo de creciente interés para los países asiáticos. Así, estos últimos incrementaron crecientemente su comercio con la región, demandando alimentos, minerales, combustibles 
y demás materias primas. Por su parte, América Latina también aumentó su demanda de importaciones de Asia, pero en este caso consumiendo productos industriales y de alto valor tecnológico. Con lo cual, el viejo patrón comercial de antaño que caracterizó a la región con las potencias desarrolladas no parece haberse roto, sino tan sólo mutado de geografía: mientras antes América Latina vendía productos primarios a Inglaterra, Europa y Estados Unidos a cambio de bienes manufacturados, ahora reproduce el mismo esquema sólo que el eje del comercio parece estar desplazándose hacia China y Asia Oriental. De esta forma, la región se mantiene estancada y en una posición de ser sólo proveedora de recursos primarios, sin hacer saltos tecnológicos, industriales o agregar valor a su producción. Es decir, continúa en una postración económica que a pesar de algunos cambios mantiene sus rasgos centrales.

Ahora bien, el caso del litio parece ofrecer una oportunidad distinta, dado que este recurso ha tendido a valorizarse y a sugerir la oportunidad no sólo de comercializarse como materia prima básica, sino también de industrializarse y de ser un eje vital en los campos tecnológicos de punta. En este sentido, es necesario tener en cuenta que el litio se ha convertido en los últimos años en un recurso de atención cada vez mayor, el cual hasta no hace mucho era un elemento poco tenido en cuenta. El mismo se usaba principalmente para la producción de cerámicas y algunos psicofármacos. No obstante, los cambios recientes en el mercado tecnológico lo han ido ubicando lentamente como un recurso estratégico de cara al futuro, el cual ya es mencionado por algunos especialistas como «el petróleo del siglo XXI».

En este caso, dadas las características del litio, su futuro lo vuelve presa de muchas especulaciones, ya que es un metal liviano que permite almacenar gran cantidad de energía y que ésta sea utilizada con suma potencia. Así, el uso para baterías de celulares, computadoras, tablets, cámaras y hasta automóviles eléctricos requieren - y eventualmente van a requerir en cantidades cada vez mayores - litio como un componente clave en su sistema energético. De allí que con sus bajos costos de explotación y virtual desarrollo hagan que su demanda con vistas al futuro sea sumamente alentadora. De este modo, se vislumbra la opción que permita eventualmente reemplazar a las energías fósiles (sobre todo el gas y el petróleo).

El objetivo de este trabajo es analizar cómo se establecen los vínculos de tenencia, producción y consumo de litio en función de los problemas que implica el neoextractivismo en Sudamérica - especialmente en Argentina- y de la creciente demanda de China y de Asia Oriental en esta región. Así, se buscará indagar las oportunidades y peligros que despierta la explotación en gran escala de este recurso y si es posible romper el patrón centro-periferia en el siglo XXI. Para hacer esto se divide el trabajo en dos partes. En la primera, se intenta señalar cómo fue la dinámica del mercado del litio desde la década de 1990 hasta la actualidad, repasando sus diversas transformaciones - el uso que se le daba, precios, principales empresas, países, etc. - y cómo operaron las mutaciones de cada caso y por qué. En la segunda parte se trata de explicar los patrones de funcionamiento del mercado del litio entre regiones, abordando cómo es el actual vínculo con respecto al litio entre Latinoamérica y Asia Oriental. Finalmente, se cierra el trabajo con algunas conclusiones al respecto. 


\section{LAS TRANSFORMACIONES DEL LITIO EN LOS ÚLTIMOS AÑOS}

El litio es el metal más liviano de todos los conocidos. Es un alcalino blando color blanco plata, que su bajo nivel atómico lo vuelve el tercer elemento de la tabla periódica, convirtiéndolo también en el sólido más liviano existente. Estas características le permiten que, con tener tan sólo la mitad de la densidad del agua, sea un excelente conductor de calor y de energía. De este modo, con todas estas peculiaridades, el litio ha sido un componente clave en diversos tipos de industrias y productos, las cuales han ido principalmente desde las cerámicas y vidrios, hasta la energía nuclear, pasando por las grasas lubricantes, los aires acondicionados, gomas sintéticas y el aluminio. Por su parte, es el principal insumo en la producción de psicofármacos y componente básico de los medicamentos más vendidos del mundo, siendo un potente estabilizador del ánimo, indispensable en tratamientos psiquiátricos de todas las patologías que más han crecido en los últimos tiempos — depresiones, insomnio, bulimia, trastornos bipolares, hiperactividad, ansiedad, drogadicción, ataques de pánico, alcoholismo, etc.- - Así, dentro de este panorama, desde la mitad del siglo XX hasta los inicios de la revolución electro-informática, en la década de 1970, el litio se explotó en cantidades modestas y estables, principalmente de las minas de petalita estadounidenses y australianas, pasando a partir de ese momento a ganar protagonismo su explotación por los salares chilenos.

Ahora bien, el mercado del litio durante los veinte años que van desde el inicio de la década de 1990 hasta 2010 sufrió transformaciones de todo tipo, afectando severamente la totalidad de las pautas con las que se había caracterizado. Tratemos de repasar estos cambios.

Para empezar, debemos decir que el litio es un elemento que se encuentra en diversas fuentes: aguas de mar, yacimientos geotérmicos, petrolíferos, salmueras, minerales rocosos, etc., distribuidos todas ellas alrededor del planeta de distintas maneras. Por lo cual, no existe un monopolio del recurso ni una sola forma de acceder a él. Sin embargo, las maneras de extraerlo de modo económicamente viable son prácticamente dos: como mineral espodumeno y a través de salmueras, donde, hasta mediados de la década de 1990, la primera forma proveía casi las dos terceras partes de la producción, sobre todo por parte de Australia, y el tercio restante, bajo la segunda forma desde Chile. Cabe destacar que en 1997 se produjo una importante modificación en el mercado del litio cuando ingresó a él la empresa chilena SQM (Sociedad Química y Minera de Chile, ex Soquimich) con una producción anual de casi 9000 toneladas de carbonato de litio. Este hecho generó dos importantes cambios. El primero fue desplomar los precios de comercialización del producto, que habían permanecido estables entre 1990 y 1996 en torno a los tres mil dólares por tonelada, haciéndolos caer un 40\% (!) y dejando su valor debajo de 1800 dólares la tonelada (ver Tabla 1). El segundo efecto se refiere a modificar la principal fuente de explotación: si en 1995 la producción de litio era explicada en un 65\% por los minerales y sólo un 35\% por salmueras, apenas una década después, en 2007, esta última forma explicaba el 86\% de lo extraído (COCHILCO, 2009, p. 35).

Sin embargo, el cambio más importante que se produjo fue otro. Nos referimos al creciente proceso de incorporación de litio para la fabricación de baterías, conocidas como Li-Ion. En efecto, durante el tramo final de la década de 1990 y principio de la siguiente, las baterías recargables reemplazaron sus principales materiales e insumos por otros, reduciendo sensiblemente sus costos de producción y adoptando un patrón tecnológico superior al vigente hasta 
Tabla 1. Recursos y reservas identificadas mundiales de litio estimadas en 2011 (en toneladas).

\begin{tabular}{lcccc}
\hline \multicolumn{1}{c}{ PAís } & $\begin{array}{c}\text { RECURSOS } \\
\text { IDENTIFICADOS }\end{array}$ & \multicolumn{3}{c}{ RESERVAS } \\
\hline Bolivia & 9.000 .000 & $27,30 \%$ & $\mathrm{~s} / \mathrm{d}$ & $\mathrm{s} / \mathrm{d}$ \\
Chile & 7.500 .000 & $22,70 \%$ & 7500000 & $57,70 \%$ \\
China & 5.400 .000 & $16,40 \%$ & 3500000 & $26,90 \%$ \\
Argentina & 2.600 .000 & $7,90 \%$ & 850000 & $6,50 \%$ \\
Australia & 630.000 & $1,90 \%$ & 580000 & $4,50 \%$ \\
Estados Unidos & 4.000 .000 & $12,10 \%$ & 38000 & $0,30 \%$ \\
Brasil & 1.000 .000 & $3,00 \%$ & 64000 & $0,50 \%$ \\
Canadá & 360.000 & $1,10 \%$ & $\mathrm{~s} / \mathrm{d}$ & $\mathrm{s} / \mathrm{d}$ \\
Zimbabue & $\mathrm{s} / \mathrm{d}$ & - & 23000 & $0,20 \%$ \\
Total & 33.000 .000 & $100,00 \%$ & 13000000 & $100,00 \%$ \\
\hline
\end{tabular}

Fuente: (Ministerio de Economía, 2011). Nota 1: Recurso identificado: es una concentración de mineral potencialmente extraíble, cuya localización, grado, cantidad y calidad son conocidas o estimadas a partir de evidencia geológica. Nota 2: Reservas: es la parte de los recursos identificados que reúne los requisitos físico-químicos mínimos para llevar a cabo prácticas de producción minera y cuya explotación económicamente viable en las condiciones actuales.

ese momento. Así, las baterías recargables más usuales eran aquellas que funcionaban en base al níquel y cadmio, aunque pronto los cambios tecnológicos hicieron que las baterías en base al litio ofreciera capacidades superiores a aquéllos: tienen un mejor trasmisor de la energía, sobre todo para almacenarla, contando con una alta densidad que facilita su recarga y distribución de manera optima, con un funcionamiento estable - sobre todo para soportar los cambios de temperatura-, resistencia a la descarga y casi sin generar residuos o pérdidas, amén de elevar sensiblemente el número de ciclos de regeneración, lo que le otorga una vida útil mucho mayor. Con lo cual, las baterías de Li-Ion son capaces de ofrecer mayor capacidad, menor peso, bajos costos, mayor potencia y sin riesgos de acción cancerígena para los usuarios como los que llegaron a ofrecer los productos en base al níquel y al cadmio. Por lo cual, los cambios operados en los insumos, producción y uso de las baterías sufrieron un importante vuelco. Así, pasó a aumentar fuertemente la demanda de litio en reemplazo del consumo de níquel y cadmio, los cuales tendieron a caer. De este modo, si las baterías en base litio eran menos del 5\% del mercado mundial en 1995, para el año 2008 eran ya el 58\% del total.

Por su parte, los cambios tecnológicos que hicieron que las baterías tuvieran como insumo fundamental al litio, también sufrieron una doble revolución interna. Por un lado, porque al comenzar el siglo XXI la revolución nanotecnológica cobró un impulso inusitado, el cual permitió que nuevos productos irrumpieran en el mercado, reemplazando por su precio y calidad a modelos que, de golpe, resultaron obsoletos. Así, productos que habían funcionado como vanguardia en la era analógica, como los walk-man, disc-man, cámaras con rollo o video cintas y celulares, dieron lugar a los reproductores de MP3, celulares y cámaras digitales, computadoras 
portátiles, e-readers, etc. Por otro lado, porque la revolución nanotecnológica fue una «revolución dentro de una revolución», ya que se modificaron radicalmente las formas de consumo y producción, consolidando la nueva era digital-informática con las telecomunicaciones, internet, nuevos servicios, precios accesibles — con tendencias a la baja - y formas dinámicas; donde el mercado de todos estos nuevos productos cobró un giro exponencial de crecimiento. De esta manera, por ejemplo, el litio usado para la fabricación de teléfonos celulares pasó de 1,8 toneladas en 1996 a 170 en 2005. Es decir, sólo en este ítem el consumo de litio se multiplicó por 94 veces en apenas 10 años. De igual modo, el consumo de litio para baterías de computadoras portátiles subió, en igual periodo, de 3,3 toneladas a 99, sufriendo un aumento de $3000 \%$. Si consideramos, además, que el uso de celulares pasó, sólo en los Estados Unidos, de 340 mil unidades en 1985 a 180 millones en 2004 y la importación de computadoras portátiles creció $1200 \%$ de 1996 a 2005, mientras que la de cámaras digitales lo hizo un 5600\% en igual plazo, podemos tener una idea del gigantesco crecimiento operado (Bruckman, 2012, p. 84).

Asimismo, con todos estos cambios, el mercado del litio volvió a sufrir transformaciones internas una vez más en muy corto tiempo. Un caso de ello fue que sus principales destinos como insumo pasaron a modificarse. Por ejemplo, en el año 1998 sus destinos se encontraban dominados para la producción de vidrios y cerámicas (destinándose el $47 \%$ del litio producido a esto), grasas lubricantes (17\%), baterías (7\%), aluminio (6\%) y aires acondicionados (5\%). Sin embargo, poco más de una década después, en 2009, la fisionomía interna de los destinos por los cuales era demandado el litio se modificó de manera notoria: las baterías prácticamente cuadriplicaron su demanda relativa total y pasaron, de tener el tercer lugar de consumo a convertirse en el principal fin con el 27\% (el resto de sus destinos, en orden de importancia, no se modificó mucho: vidrios y cerámicos, $17 \%$; grasas lubricantes, $12 \%$; aires acondicionados, 5\%; aluminio, 4\%; polímeros, 4\%; y usos farmacéuticos, 3\%) (COCHILCO, 2009 , p. 6). A su vez, todos estos cambios hicieron que, en muy poco tiempo, los precios del litio casi se triplicaran — sobre todo a partir del año 2005, cuando la producción del Salar de Atacama tuvo problemas y la demanda asiática subió mucho ya que era la principal fuente de consumo-, pasando a costar la tonelada, de un promedio cercano a los dos mil dólares, a superar los seis mil. De esta manera, aún la crisis financiera del año 2008, los valores del litio tendieron a consolidarse en niveles altos, aún cuando la producción subió mucho, ya que la demanda subió todavía más (ver Figura 1).

Sin embargo, si todos estos cambios parecen ya de por sí espectaculares, no debemos descuidar la aplicación que promete volverse la más importante de todas, convirtiendo al litio en un recurso estratégico central de cara al futuro: su uso para la fabricación de baterías de autos eléctricos. En efecto, las transformaciones tecnológicas han permitido que la fabricación de baterías Li-Ion pueda extenderse al mercado automotriz, pudiendo ofrecer una forma de almacenar energía eléctrica con niveles de potencia, autonomía y tiempo de recarga aceptables. Los automóviles eléctricos - Vehículos Eléctricos (VE), Vehículos Eléctricos Híbridos (VEH) y Vehículos Eléctricos Híbridos Plug-in o Enchufables (PHEV)requieren de un tipo especial de batería para almacenar la energía generada, ya sea por el motor a combustión interna o por otros medios. Así, con los materiales livianos, poco volumen y gran capacidad de almacenamiento, las baterías Li-Ion son las que mejor se han 
Figura 1. Evolución de los precios anuales promedios de carbonato de litio (U\$S corrientes / tonelada) (1990-2009).

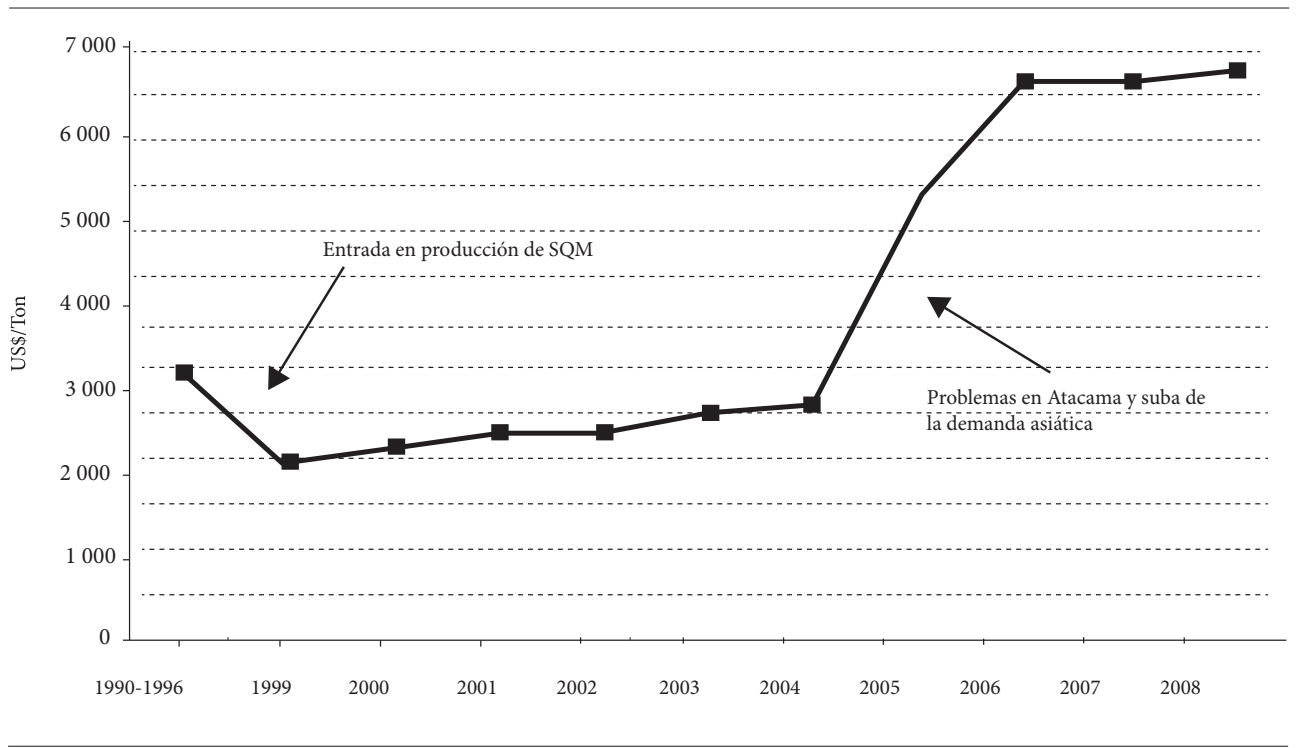

Fuente: (COCHILCO, 2009, p. 13).

adaptado a estos requerimientos, combinando el motor a combustión interna tradicional (para el caso de los híbridos) con una batería y motor eléctrico. Este tipo de automóviles ya permite alcanzar viajes que vayan de 60 a $350 \mathrm{~km}$ (dependiendo del modelo) solamente con el impulso de una batería en base al litio. A su vez, con la tecnología actual, las baterías son capaces de ser recargadas en seis minutos en cualquier enchufe doméstico de 220 voltios, alcanzando los autos velocidades máximas de $180 \mathrm{~km} / \mathrm{h}$ y una aceleración de 0 a $100 \mathrm{~km} / \mathrm{h}$ en menos de seis segundos. Muchas empresas automotrices líderes ya están disputando la vanguardia tecnológica para dominar el mercado de los autos eléctricos que, se augura con seguridad, será el dominante en el futuro. Empresas como la estadounidense General Motors, las japonesas Toyota y Nissan o las europeas Porche, Mercedes Benz y Volvo ya tienen prototipos en producción e investigación. Si bien la mayoría de los autos eléctricos actuales no utilizan baterías Li-Ion, las nuevas generaciones de estos modelos se espera que lo hagan de forma masiva. En este sentido, el mercado actual de automóviles eléctricos lentamente fue cobrando una fisionomía que proyecta ciertas cristalizaciones, sobre todo con respecto al litio y el rol central que está ocupando Asia oriental en ello. En este caso, porque la automotriz japonesa Toyota Motor Corp. ha sido hasta ahora la empresa líder en la producción de automóviles eléctricos, controlando cerca del $80 \%$ de este mercado, el cual viene dominando desde 1997 cuando sacó su primer modelo eléctrico a la venta. A su vez, sus asociaciones con otras empresas japonesas de electrónica, como Panasonic y Sanyo, le han permitido también liderar la venta de baterías eléctricas para autos. Estas condiciones le han otorgado ventajas tecnológicas de vanguardia en el mercado, 
forzando a otras automotrices a ingresar a la era eléctrica bajo su licencia Hydbrid Synergy Drive para poder producir en serie (tal fue el caso de Ford, Subaru, Mazada y Nissan). Por su parte, General Motors lanzó el Chevrolet Volt en 2012, ocupando el segundo puesto en ventas de autos eléctricos, mientras que Nissan —en alianza con Renault- piensan lanzar su vehículo eléctrico Nissan Leaf en 2015.

Del mismo modo, Volvo, Hyundai, Kia, Mercedez Benz, Seat y Tesla Motors también tienen proyectos de lanzar sus modelos eléctricos pronto (Ministerio de Economía, 2011, p. 41). En el año 2012 la venta total de automóviles eléctricos fue de 2 millones de unidades, proyectando para el año 2020 cuadriplicar ése número, arribando a los 8 millones y así representar el 10\% del mercado mundial automotriz. Para el año 2025 se espera duplicar los números previos y ocupar el $20 \%$ de la industria automotriz. Existen diversas formas de hacer las estimaciones. Puede verse tres formas de ello en (CEDHA, 201, p. 22), (FMC, 2011, pp. 9-12) y en (Ministerio de Economía, 2011, p. 42).

Estos guarismos implicarán, según una estimación realizada por Signum Box (2012), que la demanda de litio para la producción de autos sea - sólo en este ítem- de 60 y 180 mil toneladas para los años 2020 y 2025 respectivamente, teniendo en cuenta lo que demandarán los diferentes modelos de automóviles y la cantidad de ellos que saldrán a la venta - por ejemplo, la batería del Toyota Prius Plug-in requiere 3,6 kg de carbonato de litio, el Mitsubishi-iMiEV entre 10 y $15 \mathrm{~kg}$, mientras que el Tesla Roadster demandará entre $40 \mathrm{y}$ $50 \mathrm{~kg}$ por unidad-. Con lo cual, estos elementos permiten augurar un cambio de patrón energético global, realizando una transición del paso del petróleo a la energía eléctrica, convirtiéndose el litio en el insumo estratégico indispensable para llevarla a cabo.

\section{EL LITIO: ENTRE LA TENENCIA}

\section{LATINOAMERICANA Y EL ASCENSO DE ASIA ORIENTAL}

Como dijimos al comienzo del apartado anterior, la distribución del litio en el mundo guarda una forma heterogénea. Según se dijo, existen diferentes formas en las que está depositado el recurso, no sólo por tipo (salmueras, minerales, etc.), sino también por país. Además, en cada caso, deben tenerse en cuenta el grado de concentración, tasas de evaporación, cantidad y calidad, lo que hace variar sensiblemente los costos de explotación de una situación a otra. Así, debemos tener en cuenta que los recursos mundiales estimados, considerando todas las formas en las que se haya depositado el litio, acumulan cerca de 33 millones de toneladas. Sin embargo, las formas bajo las cuales el recurso es económicamente viable, dadas las tecnologías actuales, son sólo un 40\% de ese total, reduciéndose a 13 millones (ver Tabla 1) - un resumen del debate sobre cómo estimar las cantidades mundiales se puede ver en Riva Palacio (2012, p. 25-26) - . Por lo cual, no todos los países ni formas en las que se halla el recurso están en condiciones de utilizarlo, siendo un número reducido de países los únicos que pueden hacerlo.

En este sentido, hay tres datos que devienen fundamentales para analizar a los países que cuentan con el recurso. Por un lado, es que la principal promesa a futuro con respecto a la utilización del litio, gracias a convertirse en el insumo clave de los nuevos tipos de baterías, es principalmente viable a partir de la extracción del litio de las salmueras, ya que esta fuente es 
Tabla 2. Reservas mundiales de litio por tipo y país (en toneladas).

\begin{tabular}{|c|c|c|c|c|c|c|}
\hline PAÍs / FUENTE & PEGMATITAS & SALMUERAS & $\begin{array}{l}\text { SALMUERAS } \\
\text { GEOTERMA- } \\
\text { LES Y POZOS } \\
\text { PETROLÍFEROS }\end{array}$ & $\begin{array}{c}\text { ARCILLAS } \\
\text { (HECTORITA) }\end{array}$ & JADARITA & $\begin{array}{c}\text { TOTAL } \\
\text { RESERVAS }\end{array}$ \\
\hline EEUU & 2.830 .000 & 40.000 & 1.750 .000 & 2.000 .000 & - & 6.620 .000 \\
\hline Canadá & 255.600 & - & - & - & - & 255.600 \\
\hline Zimbawe & 56.700 & - & - & - & - & 56.700 \\
\hline Zaire & 2.300 .000 & - & - & - & - & 2.300 .000 \\
\hline Australia & 262.800 & - & - & - & - & 262.800 \\
\hline Austria & 100.000 & - & - & - & - & 100.000 \\
\hline Finlandia & 14.000 & - & - & - & - & 14.000 \\
\hline Rusia & 1.000 .000 & - & - & - & - & 1.000 .000 \\
\hline Serbia & - & - & - & - & 850.000 & 850.000 \\
\hline Brasil & 85.000 & - & - & - & - & 85.000 \\
\hline China & 750.000 & 2.640 .000 & - & - & - & 3.390 .000 \\
\hline Bolivia & - & $9.000 .000^{(1)}$ & - & - & - & 9.000 .000 \\
\hline Chile & - & 6.900 .000 & - & - & - & 6.900 .000 \\
\hline Argentina & - & 2.550 .000 & - & - & - & 2.550 .000 \\
\hline Total & 7.654 .100 & 21.130 .000 & 1.750 .000 & 2.000 .000 & 850.000 & 33.384 .100 \\
\hline
\end{tabular}

Fuente: COCHILCO, (2009). Nota: En la fuente original figuran 5.500.000. Lo hemos actualizado.

la más rentable para alcanzar el grado de pureza necesario. Lo cual, vuelve a acotar — una vez más- el número de países en condiciones de explotar el recurso para ése fin, reduciéndose los países prácticamente a cinco: Estados Unidos, China, Argentina, Bolivia y Chile (ver Tabla 2). Por otro lado, no es un dato menor que sólo los precios actuales han permitido que varios de estos países puedan incorporarse al mercado mundial como productores de litio, dado que el valor de la tonelada al superar los tres mil dólares - para tomar un precio de referencia- logra cubrir los costos de explotación. Es por eso que, anteriormente al año 2005, sólo el salar chileno de Atacama era el único que estaba en condiciones de ofrecer el producto; donde, cualquier descenso abrupto del precio de referencia, convertiría a muchos países y fuentes en inviables para explotar el litio en función de la calidad requerida para las nuevas tecnologías (ver Tabla 3).

Por último, y en tercer lugar, teniendo en vista todo lo anterior, es fácil percibir el lugar que le otorga el mercado mundial del litio a Latinoamérica. En efecto, Argentina, junto con Bolivia y Chile conforman lo que se ha denominado «el triángulo del litio», concentrando entre los tres países el 55\% de las reservas mundiales y cerca del 85\% de los depósitos de salmueras. Es por ello que la revista Forbes comenzó a hablar de la unión de los tres países como «la Arabia Saudita del litio», ya que guardan las reservas de materia prima para acumular la energía que se proyecta como indispensable hacia el futuro, al igual que lo fue el petróleo — según 
Tabla 3. Principales salmueras de producción de litio y su concentración, tasas de evaporación, costos unitarios y recursos identificados.

\begin{tabular}{|c|c|c|c|c|c|}
\hline PAÍs/SALAR & $\begin{array}{l}\text { CONCENTRACIÓN } \\
\text { PROMEDIO DE LITIO }\end{array}$ & $\begin{array}{c}\text { TASA DE } \\
\text { EVAPORACIÓN }\end{array}$ & $\begin{array}{l}\text { costos } \\
\text { UNIT TOT. }\end{array}$ & & $\begin{array}{c}\text { RECURSOS } \\
\text { IDENTIFICADOS }\end{array}$ \\
\hline & PPM & MM/AÑO & US\$/IB & $(\mathrm{US} \$ / \mathrm{TN})$ & TN DE LITIO \\
\hline \multicolumn{6}{|l|}{ Chile } \\
\hline Salar de Atacama & 1500 & 3700 & $0,7-1,0$ & $1543-2205$ & 6.900 .000 \\
\hline Salar de Maricunga & $400-1500$ & - & $1,2-1,5$ & $2646-3307$ & 200.000 \\
\hline \multicolumn{6}{|l|}{ Argentina } \\
\hline $\begin{array}{l}\text { Salar del Hombre } \\
\text { Muerto }\end{array}$ & 692 & 2775 & $1,1-1,3$ & $2425-2866$ & 850.000 \\
\hline Salar del Rincón & 397 & 2600 & $1,2-1,5$ & $2646-3307$ & 1.400 .000 \\
\hline Salar de Olaroz & 900 & - & $1,1-1,3$ & $2425-2866$ & 300.000 \\
\hline \multicolumn{6}{|l|}{ Bolivia } \\
\hline Salar de Uyuni & 350 & 1500 & $1,3-1,8$ & $2866-3968$ & 5.500 .000 \\
\hline Salar de Copiasa & 340 & - & - & - & 200.000 \\
\hline \multicolumn{6}{|l|}{ Estados Unidos } \\
\hline Clayton Valle & 360 & 900 & $1,1-1,3$ & $2425-2866$ & 40.000 \\
\hline Great Salt Lake & 40 & - & $1,3-1,8$ & $2866-3968$ & 520.000 \\
\hline \multicolumn{6}{|l|}{ China } \\
\hline Taijanair & 360 & 3560 & $1,1-1,3$ & $2425-2866$ & 940.000 \\
\hline Zhabuye & 1200 & - & $1,0-1,2$ & $2205-2646$ & 1.530 .000 \\
\hline Dangxiongscuo & $400-500$ & 2300 & $1,0-1,2$ & $2205-2646$ & 170.000 \\
\hline
\end{tabular}

Fuente: Ministerio de Economía (2011).

se dice- durante el siglo XX. No son pocos los analistas que se entusiasman en hablar del litio como «el petróleo del siglo XXI» o como «el oro blanco de la energía». En este sentido, Rodolfo Tecchi, quien hasta hace poco era Director de la Agencia de Promoción Científica y Tecnología del Ministerio de Ciencia de la Nación de Argentina, trató de hacer proyecciones: «Se podrían establecer mecanismos de control de la oferta de carbonato de litio, evitando distorsiones por sobreproducción que impliquen bajas en el precio internacional», las cuales afecten la rentabilidad de los tres países, atisbando que «En un futuro cercano y con una producción plena, Bolivia, Argentina y Chile van a manejar el mercado del litio. Podrían hacerlo en una suerte de OPEP» (Clarín, junio 29 de 2011). Con lo cual, la búsqueda de mecanismos comunes de defensa de los intereses de la región devendrá un tema clave, por eso no es descabellado augurar por la creación de organismos supranacionales al respecto. Un suceso en esta dirección fue el llamado a conformar un «G-10 de la energía» en Bolivia en mayo de 2014, al cual los tres países con litio en la región acudieron. Como dijo el presidente de YPF (Yacimientos Petrolíferos Fiscales, de Argentina), Miguel Galuccio, «si tener el dominio de la 
Tabla 4. Exportaciones de carbonato de litio en valores totales (en millones de dólares) y por país (en \%) (2001-2013).

\begin{tabular}{|c|c|c|c|c|c|c|c|c|c|c|c|c|c|}
\hline AÑO & 2001 & 2002 & 2003 & 2004 & 2005 & 2006 & 2007 & 2008 & 2009 & 2010 & 2011 & 2012 & 2013 \\
\hline \multicolumn{14}{|c|}{ EXPORTACIÓN MUNDIAL TOTAL (VALOR) } \\
\hline Total & 85 & 89 & 108 & 133 & 165 & 227 & 321 & 358 & 219 & 298 & 343 & 370 & 355 \\
\hline \multicolumn{14}{|c|}{ EXPORTACIÓN MUNDIAL POR PAÍS (EN \%) } \\
\hline Chile & 60,90 & 62,38 & 59,90 & 56,30 & 57,10 & 54,30 & 58,70 & 61,10 & 51,50 & 58,40 & 59,40 & 66,72 & 63,50 \\
\hline Argentina & 2,90 & 1,18 & 5,58 & 9,77 & 10,00 & 11,50 & 9,24 & 13,90 & 18,40 & 15,90 & 11,30 & 11,10 & 11,20 \\
\hline Bélgica & 7,11 & 8,33 & 7,24 & 7,53 & 7,92 & 8,84 & 9,41 & 5,68 & 7,81 & 7,28 & 5,80 & 6,43 & 10,00 \\
\hline China & 3,34 & 2,93 & 2,56 & 2,43 & 3,45 & 7,55 & 6,51 & 4,52 & 6,29 & 6,63 & 10,00 & 5,42 & 2,61 \\
\hline Alemania & 6,49 & 5,96 & 7,34 & 6,50 & 6,51 & 6,34 & 5,89 & 5,08 & 6,59 & 5,00 & 6,26 & 4,26 & 6,27 \\
\hline EE. UU & 12,90 & 14,40 & 11,80 & 11,40 & 10,50 & 6,57 & 5,56 & 6,40 & 4,92 & 4,05 & 3,72 & 3,47 & 3,37 \\
\hline $\begin{array}{l}\text { Corea } \\
\text { del Sur }\end{array}$ & 0 & 0,03 & 0,26 & 0,04 & 0,01 & 0 & 0 & 0,10 & 0,52 & 0,89 & 0,46 & 0,56 & 0,31 \\
\hline Eslovenia & 1,20 & 0,85 & 0,81 & 0,95 & 0,85 & 0,66 & 0,57 & 0,58 & 1,29 & 0,66 & 0,57 & 0,50 & 0,37 \\
\hline Holanda & 0,74 & 0,40 & 0,34 & 0,35 & 0,46 & 0,48 & 0,76 & 0,60 & 0,76 & 0,34 & 0,57 & 0,47 & 0,48 \\
\hline $\begin{array}{l}\text { Reino } \\
\text { Unido }\end{array}$ & 0,50 & 0,33 & 0,49 & 0,33 & 0,44 & 0,60 & 0,36 & 0,19 & 0,48 & 0,16 & 0,13 & 0,22 & 0,17 \\
\hline Otros & 3,88 & 3,17 & 3,60 & 4,38 & 2,65 & 3,10 & 2,99 & 1,81 & 1,36 & 0,62 & 1,69 & 0,79 & 1,68 \\
\hline Total & 100 & 100 & 100 & 100 & 100 & 100 & 100 & 100 & 100 & 100 & 100 & 100 & 100 \\
\hline
\end{tabular}

Fuente: Elaboración propia en base datos TRADEMAP.

Tabla 5. Exportación de carbonato de litio argentino por país de destino (en \%) (2003-2013).

\begin{tabular}{|c|c|c|c|c|c|c|c|c|c|c|c|}
\hline PAÍS & 2003 & 2004 & 2005 & 2006 & 2007 & 2008 & 2009 & 2010 & 2011 & 2012 & 2013 \\
\hline EE. UU. & 89,18 & 93,09 & 63,74 & 88,78 & 95,33 & 88,17 & 87,98 & 76,30 & 79,15 & 57,85 & 51,06 \\
\hline China & 0 & 0,63 & 25,16 & 6,5 & 0,69 & 2,68 & 9,94 & 10,04 & 6,02 & 19,09 & 32,79 \\
\hline $\begin{array}{l}\text { Corea } \\
\text { del Sur }\end{array}$ & 0 & 0 & 0 & 0 & 0 & 0 & 0 & 0 & 1,03 & 1,76 & 4,49 \\
\hline Bélgica & 0 & 0 & 0 & 0 & 0 & 0 & 0 & 1,09 & 1,37 & 1,93 & 4,26 \\
\hline Japón & 10,82 & 6,28 & 11,1 & 4,71 & 3,97 & 0 & 2,06 & 11,34 & 11,7 & 16,84 & 4,03 \\
\hline Otros países & 0 & 0 & 0 & 0 & 0 & 9,15 & 0,02 & 0,85 & 0,66 & 2,51 & 3,37 \\
\hline Total & 100 & 100 & 100 & 100 & 100 & 100 & 100 & 100 & 100 & 100 & 100 \\
\hline
\end{tabular}

Fuente: Elaboración propia en base a datos de TRADEMAP. 
energía es poder político, agruparse y encontrar sinergias es todavía tener más poder», puntualizando que aquellos países que tienen poder político son aquellos que «dominan sus recursos naturales» (Página 12, mayo 16 de 2014).

Así, por su parte, se entiende que los cambios tecnológicos y económicos son una oportunidad que los países latinoamericanos no pueden desaprovechar, no sólo como fuente de divisas y rentabilidad excepcional, sino también, yendo todavía por un paso más, al industrializar el recurso e -incluso - llegar a fabricar las baterías localmente, incorporando un alto valor agregado y tecnológico. Como manifestó Sergio Echebarrena, presidente de la Cámara Argentina de Proveedores de la Industria Petroenergética (CAPIPE), con respecto a la gestión del recurso:

Tener el recurso y tratar de aumentar las regalías es una mirada. Creo que no se trata de pertenecer a una OPEP del litio sino de ser parte de los que tienen la tecnología y de cómo utilizar el recurso para generar la industria. [...] La industria argentina está en condiciones de incorporarse a la cadena de valor, para que el litio no salga a granel y sin escalas sino en forma de baterías. (Sevares y Krzemien, 2012, p. 142)

Con respecto a esto último, tratemos de abordar cómo se da la relación entre países exportadores de litio, los consumidores del mismo y aquellos quienes lo elaboran bajo forma de pilas y baterías. Si observamos la información provista por Trademap hasta el momento —organismo dependiente de Naciones Unidas-, en la Tabla 4 notaremos que los valores exportados de carbonato de litio entre los años 2001 y 2013 han crecido fuertemente, pasado de casi 85 mil dólares a 370 mil de un extremo a otro de la serie. Sin embargo, notaremos también que existen dos periodos de exportación bien marcados. Por un lado, una tendencia de aumento sostenido de las exportaciones entre los años 2001 y 2008, que ofreció una tasa de crecimiento anual de $22 \%$, dado principalmente por la producción latinoamericana. Sin embargo, tras el estallido de la crisis en 2008, que hizo disminuir mucho el comercio mundial, produjo un abrupto descenso de los valores exportados de litio, con una caída de casi el 40\% por esto. Así, a partir del año 2009 los números exportados volvieron a crecer hasta 2012, casi al mismo ritmo que antes, hasta recuperarse totalmente, retornando a los valores previos. A su vez, si tenemos en cuenta la performance ofrecida por la Argentina, vemos que es el país que más hizo crecer sus exportaciones en todo el periodo 2001-2013 (a una tasa del 29\% anual, cuando el mercado mundial aumentó al $14 \%$ en igual plazo). Por su parte, el destino más importante del carbonato exportado por el país fueron - de lejos - los Estados Unidos, aunque en los últimos años ha logrado desconcentrar las ventas. Así, con el ascenso del consumo de Japón y la demanda sostenida de China, permitió que la diversificación de los países compradores del litio argentino mejorara bastante (ver cuadro 5), siendo Asia Oriental el responsable del 40\% de sus ventas en 2013.

En este sentido, el veloz crecimiento argentino le permitió ir consolidando su lugar como segundo exportador mundial de carbonato de litio durante los años 2001-2013, aunque muy atrás de Chile - que explica por sí solo dos tercios del total-, pero concentrando entre ambos países casi el 80\% del negocio de exportación. Con lo cual, se confirman las premisas que indican que el cono sur latinoamericano podría aprovechar su supremacía sobre el mercado, sobre todo cuando también se agregue Bolivia como país exportador, para aplicar políticas de defensa de los precios de sus productos y buscar aumentar su valor agregado. 
Tabla 6. Importaciones de carbonato de litio en valores totales (en millones de dólares) y por país (en \%) (2001-2013).

\begin{tabular}{|c|c|c|c|c|c|c|c|c|c|c|c|c|c|}
\hline AÑO & 2001 & 2002 & 2003 & 2004 & 2005 & 2006 & 2007 & 2008 & 2009 & 2010 & 2011 & 2012 & 2013 \\
\hline \multicolumn{14}{|c|}{ IMPORTACIÓN MUNDIAL TOTAL (VALOR) } \\
\hline $\begin{array}{c}\text { TOTAL } \\
\text { (EN US\$) }\end{array}$ & 77 & 86 & 103 & 132 & 161 & 231 & 329 & 353 & 239 & 298 & 383 & 423 & 354 \\
\hline \multicolumn{14}{|c|}{ IMPORTACIÓN MUNDIAL POR PAÍS (EN \%) } \\
\hline Japón & 18,50 & 19,10 & 21,80 & 21,00 & 17,00 & 27,10 & 26,10 & 24,60 & 21,70 & 25,90 & 21,10 & 16,50 & 12,54 \\
\hline $\begin{array}{l}\text { Corea } \\
\text { del sur }\end{array}$ & 3,65 & 2,96 & 3,57 & 3,87 & 4,21 & 4,29 & 6,82 & 9,05 & 13,30 & 13,70 & 13,80 & 16,10 & 19,56 \\
\hline China & 11,20 & 9,78 & 11,00 & 12,30 & 13,7 & 10,30 & 6,72 & 6,57 & 5,27 & 9,51 & 8,77 & 14,80 & 19,41 \\
\hline EE. UU & 20,90 & 19,20 & 18,50 & 21,30 & 18,4 & 17,30 & 17,30 & 20,60 & 18,20 & 14,4 & 15,20 & 13,70 & 13,58 \\
\hline Alemania & 10,00 & 11,20 & 11,70 & 10,80 & 11,00 & 10,2 & 10,00 & 9,15 & 9,37 & 9,86 & 10,90 & 11,70 & 2,91 \\
\hline Bélgica & 8,00 & 8,28 & 6,10 & 7,22 & 7,97 & 8,06 & 8,79 & 7,03 & 7,86 & 6,06 & 8,66 & 7,43 & 9,37 \\
\hline Francia & 3,43 & 4,48 & 3,55 & 2,96 & 2,75 & 2,55 & 2,85 & 2,62 & 3,45 & 2,58 & 2,97 & 2,54 & 2,59 \\
\hline Canadá & 3,05 & 2,98 & 2,54 & 2,17 & 2,32 & 2,39 & 2,85 & 3,12 & 2,53 & 2,18 & 1,80 & 1,95 & 2,51 \\
\hline Rusia & 4,54 & 4,16 & 2,88 & 3,61 & 3,80 & 1,21 & 1,20 & 1,23 & 0,83 & 0,98 & 2,27 & 1,91 & 1,80 \\
\hline Holanda & 2,13 & 2,06 & 1,96 & 1,69 & 1,22 & 1,91 & 2,59 & 1,21 & 0,83 & 0,87 & 1,21 & 1,81 & 1,43 \\
\hline España & 1,62 & 1,47 & 1,32 & 1,16 & 1,83 & 1,52 & 1,28 & 2,33 & 2,09 & 1,99 & 2,02 & 1,80 & 2,36 \\
\hline Turquía & 0,52 & 0,59 & 0,81 & 0,84 & 0,85 & 1,03 & 1,49 & 1,13 & 1,54 & 1,34 & 1,21 & 1,45 & 2,38 \\
\hline Italia & 3,88 & 3,70 & 3,63 & 2,73 & 5,13 & 3,48 & 2,42 & 2,39 & 2,80 & 1,76 & 1,67 & 1,28 & 1,88 \\
\hline Otros & 8,44 & 9,83 & 10,4 & 8,12 & 9,59 & 8,50 & 9,43 & 8,91 & 10,00 & 8,77 & 8,31 & 6,84 & 7,68 \\
\hline Total & 100 & 100 & 100 & 100 & 100 & 100 & 100 & 100 & 100 & 100 & 100 & 100 & 100 \\
\hline
\end{tabular}

Fuente: Elaboración propia en base a datos de TRADEMAP.

Con respecto a la importación del carbonato de litio, la situación se modifica. En principio, porque se registra una distribución más atomizada entre los países compradores de carbonato de litio que la ofrecida cuando se trata de vender. A su vez, porque el consumo de carbonato de litio se explica, prácticamente en su totalidad, por los países con mayor grado de desarrollo. Es decir, puede percibirse que los países centrales, por más que puedan ofrecer ciertas cantidades de exportación de litio, son realmente quienes lo consumen sin excepción, no existiendo países periféricos entre los demandantes - es preciso tener en cuenta que los países desarrollados que exportan litio muchas veces no lo producen dentro de sus territorios geográficos, sino que registran las exportaciones a través de las empresas mineras de su nacionalidad, pero que explotan el recurso en otra parte-. Por ejemplo, mientras Bélgica, China, Alemania, EE. UU. y Corea del Sur exportaron, en conjunto, el 20\% del valor del carbonato del litio mundial en 2013, ese mismo año exportaron más del triple de lo importado, con casi el 68\% del consumo total (ver Tabla 6). Con lo cual, vemos que con el caso del litio no se rompe la lógica global aplicada a la minería: la misma se consume en el centro, pero en su mayoría es explotada 
Tabla 7. Exportaciones de pilas y baterías de pilas eléctricas de litio en valores totales (en miles de dólares) y por país (en \%) (2001-2013)

\begin{tabular}{|c|c|c|c|c|c|c|c|c|c|c|c|c|c|}
\hline & 2001 & 2002 & 2003 & 2004 & 2005 & 2006 & 2007 & 2008 & 2009 & 2010 & 2011 & 2012 & 2013 \\
\hline \multicolumn{14}{|c|}{ EXPORTACIÓN MUNDIAL TOTAL (VALOR) } \\
\hline $\begin{array}{l}\text { TOTAL } \\
\text { (EN US\$) }\end{array}$ & 715 & 776 & 1044 & 1170 & 1129 & 1157 & 1233 & 1468 & 1485 & 1815 & 1871 & 1965 & 387 \\
\hline \multicolumn{14}{|c|}{ EXPORTACIÓN MUNDIAL POR PAÍS (EN \%) } \\
\hline EE. UU. & 15,30 & 14,80 & 13,80 & 15,30 & 20,60 & 24,10 & 22,70 & 21,90 & 23,60 & 20,10 & 19,00 & 18,90 & 19,20 \\
\hline Singapur & 4,29 & 5,02 & 8,20 & 8,32 & 6,08 & 6,03 & 6,78 & 9,50 & 13,40 & 12,40 & 12,80 & 14,80 & 16,50 \\
\hline $\begin{array}{l}\text { Hong Kong } \\
\text { (China) }\end{array}$ & 3,98 & 4,40 & 4,30 & 10,10 & 13,80 & 9,95 & 7,98 & 7,71 & 7,60 & 8,92 & 8,92 & 10,50 & 1,29 \\
\hline Japón & 29,20 & 26,40 & 17,70 & 15,10 & 13,50 & 14,60 & 14,80 & 12,40 & 9,49 & 10,00 & 9,83 & 9,19 & 7,66 \\
\hline China & 1,46 & 1,97 & 3,72 & 4,21 & 4,87 & 3,86 & 5,07 & 5,04 & 5,12 & 5,60 & 6,16 & 6,32 & 8,62 \\
\hline Indonesia & 1,82 & 2,27 & 1,97 & 2,32 & 3,18 & 4,08 & 3,83 & 3,29 & 3,43 & 4,25 & 5,45 & 5,52 & 7,81 \\
\hline Canadá & 0,52 & 0,87 & 0,55 & 1,00 & 1,57 & 2,02 & 2,67 & 2,25 & 1,65 & 1,47 & 2,97 & 4,96 & 4,81 \\
\hline Alemania & 7,70 & 7,61 & 5,85 & 7,83 & 7,25 & 6,77 & 6,38 & 5,41 & 5,44 & 4,65 & 5,64 & 4,95 & 6,23 \\
\hline Francia & 6,38 & 7,08 & 5,93 & 6,18 & 5,31 & 6,23 & 6,00 & 5,83 & 5,25 & 4,62 & 4,96 & 4,85 & 5,11 \\
\hline Reino Unido & 3,10 & 2,47 & 3,35 & 4,41 & 4,21 & 4,38 & 4,34 & 4,74 & 4,20 & 3,90 & 4,45 & 3,87 & 3,63 \\
\hline Bélgica & 4,79 & 6,82 & 5,94 & 5,29 & 4,67 & 3,66 & 3,08 & 3,07 & 2,82 & 2,66 & 2,90 & 3,17 & 3,25 \\
\hline Holanda & 1,42 & 1,59 & 1,82 & 1,29 & 1,10 & 1,06 & 2,99 & 3,21 & 2,15 & 2,25 & 2,66 & 2,58 & 2,73 \\
\hline Corea del Sur & 1,01 & 1,30 & 1,04 & 1,40 & 1,40 & 1,40 & 1,90 & 2,93 & 2,34 & 1,93 & 2,03 & 2,39 & 2,76 \\
\hline Suiza & 1,83 & 1,72 & 1,69 & 2,07 & 2,11 & 3,35 & 3,77 & 3,66 & 2,79 & 2,93 & 2,25 & 0,99 & 1,01 \\
\hline México & 4,59 & 1,57 & 0,91 & 0,47 & 0,46 & 0,24 & 0,34 & 0,23 & 0,76 & 0,94 & 0,70 & 0,93 & 0,71 \\
\hline Otros & 12,50 & 13,98 & 23,07 & 14,65 & 9,77 & 8,09 & 7,34 & 8,73 & 9,84 & 13,30 & 9,15 & 6,04 & 8,54 \\
\hline Total mundial & 100 & 100 & 100 & 100 & 100 & 100 & 100 & 100 & 100 & 100 & 100 & 100 & 100 \\
\hline
\end{tabular}

Fuente: Elaboración propia en base a datos de TRADEMAP.

en los países pobres y subdesarrollados, manteniendo el clásico esquema dependentista, que no parece haberse roto. Por su parte, ha sido la fuerte demanda por el consumo de Corea del Sur y China —amén la de Japón—, la que le ha permitido a los países asiáticos explicar por sí solos más del 50\% del consumo mundial de litio, señalándonos el peso fundamental que están ocupando estos países como motores económicos en casi todos los mercados. Por lo cual, si los países latinoamericanos son los principales vendedores de litio, son los países asiáticos los que se están convirtiendo en sus principales clientes.

De tal manera, la premisa de industrializar el litio y producir baterías debe contemporizar que, actualmente, la fabricación de baterías tiene como principal núcleo al Sudeste asiático. Así, Japón y Corea del Sur han sido los países pioneros en la investigación y desarrollo de este tipo de producción, mientras que China ingresó al mercado sólo como productor de pilas y baterías de menor costo y calidad, pero logrando tener más de 100 fabricantes locales 
(Ministerio de Economía, 2011, p. 10). El carbonato de litio importado por estos tres países del Oriente asiático, según la Tabla 6, sumó, como ya dijimos, el 51,5\% del total mundial en 2013, exportando los mismos, en igual año, casi el 19\% de las pilas y baterías de pilas eléctricas de litio, pero llegando al 45\% si agregamos a los países que funcionan como re-exportadores de aquellos muchas veces por cuestiones fiscales (Singapur, Hong Kong e Indonesia) (ver Tabla 7). Es decir, esos tres países consumen casi la mitad del carbonato de litio mundial para exportar casi igual porcentaje de las baterías y pilas de baterías eléctricas del mundo, ocupando un lugar cada vez más destacado en el mercado: su participación creció del $40 \%$ a casi el 50\% entre 2001 y 2013, cuando la producción mundial casi se triplicó en ésos años (lo cual indica que tuvieron un crecimiento más aprisa que el ofrecido por el mercado mundial, liderando así su expansión). A su vez, si la oferta de la producción de pilas y baterías es relativamente acotada, esto se reduce aún más si se considera a la chance de fabricar la totalidad de los componentes técnicos.

Como indicó la Ingeniera Juana Olivares, encargada de dirigir una fabrica prototipo en Bolivia que intenta la producción local de las baterías explicando la dificultad por el momento de una producción $100 \%$ nacional, «de hecho ningún país tiene esa capacidad, incluso China debe importar la membrana separadora de Japón, que es el único país que cuenta con ese material y viene desarrollando su tecnología desde hace más de 20 años» (El Deber, febrero 17 de 2014). Es por eso que para los países latinoamericanos la posibilidad de una fabricación plena de la batería aparece como un sueño lejano. Para el caso argentino, por ejemplo, hacerlo hoy sería meramente cumplir un papel de ensamblaje, ya que el 80\% de los componentes necesarios de la batería deberían producirse afuera. El presidente de la Cámara Minera de Salta, Facundo Huidobro, piensa que es mejor dejar de destinar tiempo y dinero a una meta casi imposible, y conformarse con producir litio de manera internacionalmente competitiva, sugiriendo seguir el modelo liberal chileno de explotación: «Chile tiene más de 30 años de explotación en litio y nunca pensó en baterías» (Gacetilla del Área Minera, 2011, p. 8). Así, en los países de la región, sobre todo en Argentina y Bolivia - que tienen perspectivas más ambiciosas sobre la industrialización, la tecnología y la agregación de valor-, las aguas están divididas entre aquellos que apuestan a un desarrollo local que persiga la chance de fabricar la batería y aquellos que creen que es un camino casi imposible, y que sólo traerá perjuicios y el derroche de recursos en el largo plazo (Gacetilla del Área Minera, 2011) — para las diferentes estrategias y ambiciones con respecto al litio entre Argentina, Bolivia y Chile, ver CEDHA (2012, pp. 4051) y Sevares y Krzemien (2012, pp. 134-136) -.

En otro orden, las que despuntan como actores claves del proceso hacia el futuro son las empresas automotrices. En este caso, con el virtual agotamiento del petróleo, el encarecimiento de sus costos y/o las presiones para pasar a un paradigma de «energías verdes», las automotrices se han lanzado a la carrera de liderar el nuevo proceso de fabricación de autos eléctricos. Así, en el año 2007 se produjeron 500 mil unidades de estos, cuando la producción mundial total de automóviles fue en ése año de 73 millones de unidades (COCHILCO, 2009: 7). Es decir, una porción mínima que apenas cubrió el 0,68\% del mercado, aunque como ya dijimos los números de a poco han ido creciendo. En los países centrales ninguno de sus gobiernos quiere quedar atrás de lo que se presenta como el futuro del mercado automotriz, ya que los beneficios 
Tabla 8. Tipo de batería según tipo de auto y la incidencia del litio en el costo

\begin{tabular}{lccc}
\hline \multicolumn{1}{c}{ TIPO DE BATERÍA SEGÚN TIPO DE AUTO } & EV & PHEV & HEV \\
\hline Capacidad batería & $25 \mathrm{kwh}$ & $12 \mathrm{kwh}$ & $2 \mathrm{kwh}$ \\
Cantidad de carbonato de litio requerido & $15 \mathrm{~kg}$ & $7,5 \mathrm{~kg}$ & $1,5 \mathrm{~kg}$ \\
Costo del litio total requerido $(\approx 6$ U $\$ \mathrm{~s} / \mathrm{kg})$ & US\$ 90 & US $\$ 45$ & US\$ 10 \\
Precio final de la batería $(\approx 700$ US\$ $/ \mathrm{kmh})$ & US\$ 17.500 & US\$ 9000 & US\$ 1400 \\
Incidencia del litio en el costo unitario final & $0,51 \%$ & $0,50 \%$ & $0,71 \%$ \\
\hline
\end{tabular}

tecnológicos, laborales, industriales y económicos de la producción de automóviles son inmensos, mucho más combinados con la fuerza que implica dominar las nuevas tecnologías.

Es necesario aclarar que la minería del litio no funciona como la minería metalífera tradicional, sobre todo del oro, con voladuras de montañas, ni con el uso masivo de explosivos y cianuro. Sin embargo, sí se acerca en un aspecto sumamente cuestionado: el uso masivo del agua en lugares semidesérticos y con problemas de sequía. Como explica el geólogo Fernando Días: «Puede estimarse que por cada tonelada de litio extraída se evaporan alrededor de dos millones de litros de agua, clara evidencia de que la minería del litio en salares es una minería del agua». (No a la mina, 2012).

Sin embargo, por más que se puedan desarrollar las nuevas tecnologías, el futuro del litio no puede ligarse exclusivamente a esto. Si bien el crecimiento de la industria de autos eléctricos puede convertirse en su motor más dinámico, no debe exagerarse el rol que cumple el litio, ya que éste es bastante paradójico. Por un lado, porque aunque el litio sea un componente central de la industria, volviéndose irremplazable — no es casualidad que el tipo de batería que se piensa usar se llame, justamente, Li-ion-, también es verdad que el peso de la materia prima en la cadena de valor automotriz es casi nulo. En efecto, para producir una batería eléctrica para autos, bajo la tecnología y valor actuales, se requiere entre 7 y 15 kilos de litio, siendo esto un costo que oscila, apenas, entre los 42 y 90 dólares por vehículo. No obstante, el valor final de una batería se encuentra entre los 8 y 18 mil dólares, volviéndose el litio, en tanto materia prima, una proporción cercana al 0,5\% del total (ver Tabla 8). A su vez, si se tiene en cuenta que las baterías son tan sólo una parte del total del costo de un automóvil, el peso que ocupa el litio en el valor final es, entonces, muy bajo. Es por eso que a las automotrices no parece preocuparles el precio del recurso, sino simplemente asegurarse una pequeña provisión de él hacia futuro. En este caso, las empresas no aspiran a tener un monopolio de la materia prima o a estar intranquilas por la evolución de su precio, ya que - como vimos- es muy poco lo que requieren y menor aún la capacidad del insumo de influir en el precio final de los automóviles. Así, la mayoría de las empresas - ya sean europeas, asiáticas o estadounidenses- han decidido utilizar como principal estrategia asociarse con diversas compañías mineras - en general trasnacionales-, financiando sus proyectos, exploraciones y explotaciones, con el único fin - posteriormente- de acceder de un modo seguro al recurso por largos periodos que van desde los 20 a los 50 años. Porque es obvio que ninguna empresa va a desarrollar un plan de reconversión tecnológica multimillonario, de largo plazo, sin tener la confianza 
de la provisión de los insumos que ésta implique (Clarín, marzo 21 de 2010). En el caso argentino, este tipo de estrategia de simple resguardo del abastecimiento de litio por parte de las empresas de Asia Oriental se muestra de manera plena. Así, la automotriz japonesa Toyota se asoció con la minera australiana Orocobre Ltd. para explotar el Salar de Olaroz (provincia de Jujuy), Magna y Mitsubishi (también de Japón) lo hicieron en el Salar de Cauchari (provincia de Salta) con la minera canadiense Lithium Americas, mientras que la automotriz coreana Kores lo hizo con Lithium One (minera canadiense-china) para explotar el yacimiento Sal de Vida en Catamarca (Sevares y Krzemien, 2012, p. 141). Con lo cual, las automotrices, si bien son un agente fundamental de la cadena de comercialización, en ningún caso reclaman la exclusividad o control del litio, ya que sus contratos con las mineras sólo se refieren a una parte de la producción total lograda, estando éstas en libertad de vender el producto cómo y a quienes quieran, sin tampoco mostrar - como dijimos- preocupación por los aumentos que pudiera sufrir el precio de cotización. Es decir, a los países latinoamericanos no les es suficiente con contar con el recurso para controlar la cadena de valor y, en consecuencia, iniciar un proceso de desarrollo industrial autónomo, por lo que la tarea dependerá más de la capacidad de sus gobiernos de realizar políticas al respecto que de la simple tenencia del litio. Como señaló Tecchi con vistas a esto:

Si se repiten las conductas que se siguieron con la explotación de otros recursos naturales, no llegará el desarrollo a nivel local. Si lo único que queda de la explotación del litio son las regalías mineras y unos pocos puestos de trabajo, va a ser preferible preservar los salares para alguna otra actividad futura. (Sevares y Krzemien, 2012, p. 144)

\section{CONCLUSIONES}

A lo largo de este trabajo hemos intentado recorrer diferentes aspectos que tienen que ver con la explotación, comercialización y consumo del litio en el mundo, enfocándonos especialmente en las consecuencias para los países de Sudamérica y su relación con el oriente de Asia. En él tratamos de repasar pasado, presente y futuro de un recurso que ha cobrado vuelo propio en los últimos años, concentrando una atención cada vez mayor. Como vimos, los cambios en el mercado del litio han sido muy grandes, prometiendo volverse mayores todavía hacia adelante. Sin embargo, como señalamos, a pesar de las veloces transformaciones operadas el litio no está destinado a ser el sustituto del petróleo ni tampoco a volverse un recurso invaluable. Igualmente, y tomando estas salvedades, tampoco son menores las oportunidades que ofrece con vistas a aprovecharlo, por ejemplo, como el primer paso de un proceso industrial y tecnológico cada vez mayor, pero tampoco a descuidar los peligros que puede implicar su explotación a gran escala.

No existe en la actualidad perspectiva de una explotación del litio en forma sustentable. La minería, por definición, es una actividad contaminante que destruye la naturaleza y el medioambiente. Por lo cual, depender de ella únicamente como fuente de divisas es adoptar un patrón de acumulación por extracción que condena a los países al despojo. Así, de no existir una intervención de forma activa y dinámica por parte de los gobiernos las perspectivas abiertas por la posesión de un recurso de valorización creciente como es el litio 
se presentarán como un riesgo, no sólo ecológico o territorial, sino también productivo al reprimarizar las economías. Los modelos extractivistas meramente proveedores de divisas no generan «derrames» sobre el territorio sobre el cual se realizan. Por lo cual, por el mero anhelo de obtener divisas de manera rápida y lucrativa se puede condenar a un país a la postración. Porque la minería, como se dice popularmente, «es pan para hoy pero hambre para el mañana». La nueva división internacional del trabajo entre zonas y países industriales, con capacidad tecnológica de vanguardia, y aquellos países rezagados, destinados simplemente a producir la materia prima de los insumos fabricados en el centro, parece no haber roto los viejos patrones de antaño, sino simplemente a mutar el rol ocupado, por ejemplo, de Gran Bretaña en el siglo XIX o Estados Unidos en el XX, por el de China y Asia Oriental en el siglo XXI, cayendo en un modelo neoextractivista que igualmente no evade sus límites. En este caso, hablamos de neoextractivismo en la medida en que la explotación primaria tradicional en América Latina (minería, petróleo, agronegocio, etc.) continua siendo promovida con el fin de generar una mayor exportación, aunque ahora buscando también nuevos tipos de «derrames», como pueden ser una mayor integración regional, algunos derivados productivos y - sobre todo- que los Estado puedan captar parte de la renta extractiva para aplicar medidas distributivas — con respecto a los «nuevos derrames» buscados, éstos, al igual que antaño, son muy limitados o casi nulos y a veces contraproducentes. Con respecto a la integración regional, y a pesar de ciertos avances en Sudamérica, gracias al vínculo de América Latina con China se está generando la tendencia opuesta en algunos aspectos. Por ejemplo, en el caso de Brasil y Argentina, países que han tendido a afianzarse mucho entre sí durante las últimas décadas, aumentaron su competencia entre ellos para ganar el acceso al mercado chino (y a exportar hacía allí) pero al costo de suscribir a convenios comerciales desventajosos con el gigante asiático, mientras que también han tendido a reemplazarse mutuamente como proveedores de bienes manufacturados de alto, medio y bajo valor tecnológico por aquellos provistos por China, desdibujando así la integración lograda (Slipak, 2014b). Con vistas a la captación y distribución de rentas, ya sea por el extractivismo tradicional o por el de nuevo tipo, puede encontrarse una buena crítica en Acosta (2011) y Féliz (2011) a las consecuencias que genera y sus costos ocultos, como también un buen resumen de los debates al respecto en Gudynas (2011). Por último, en relación a los «derrames» productivos, inmediatamente se señalan sus límites-.

En este sentido, la explotación del litio a diferencia de otro tipo de minería no implica sólo la extracción de una materia prima, sino que también ofrece la posibilidad a los países que lo poseen de montarse sobre el proceso de cambio tecnológico y de la transformación de la matriz energética actual. Aunque, de igual modo, los problemas y dificultades para aprovechar esto no son menores, lo cual nos señala que los países sudamericanos están amenazados con mantener sólo un rol menor en este nuevo proceso, probablemente casi de saqueo como antaño, en el que obtengan meramente unas pocas divisas por sus recursos y asuman los peligrosos costos de quedarse con los pasivos ambientales que la minería conlleva. Así, los países de la región terminarían en los hechos, irónicamente, financiando ellos el nuevo desarrollo de los países del centro y la consolidación de Asia Oriental como eje económico mundial, puesto que a largo plazo pueden quedarse sólo con tierras 
contaminadas, poblaciones invadidas y desplazadas, ambientes intoxicados, desperdicio de aguas y territorios arrasados mientras el centro, nuevamente, continua con su dominio planetario a costas de los países subdesarrollados. Porque en contraste con los años 80 y 90 , al comenzar el siglo XXI las economías latinoamericanas se vieron enormemente favorecidas por los altos precios internacionales de los productos primarios (commodities), empujado esto principalmente por el ascenso económico chino y su voraz demanda, en lo que la totalidad de los gobiernos latinoamericanos más allá de su signo ideológico se lanzaron a aprovechar la coyuntura favorable, estableciéndose con este proceso lo que algunos autores han denominado el «consenso de los commodities» (Svampa, 2013).

Así, en los últimos tiempos se tendió a subrayar las ventajas comparativas del boom comercial y los beneficios ligados a los niveles de captación de divisas, inversiones, préstamos y exportaciones que significó, pero al riesgo de descuidar las consecuencias que el proceso pudiera implicar. Los especialistas argentinos Svampa y Slipak (2015, p. 20) agudamente se han encargado de señalar al respecto: «Las inversiones que la región latinoamericana recibe de China, no tienden a desarrollar capacidades locales, ni actividades intensivas en conocimiento u encadenamientos productivos. La localización de las empresas chinas, como los préstamos contra commodities, tiende a potenciar las actividades extractivas en detrimento [de] aquellas con mayor valor agregado, lo cual refuerza el efecto reprimarizador que nuestras economías viven bajo lo que hemos llamado el 'Consenso de los Commodities'». Un amplio y detallado análisis al respecto se encuentra en Cepal (2010, p. 103-136).

Por lo que se puede estar montando una peligrosa trampa que luego resulte muy difícil romper, ya que las exportaciones latinoamericanas hacia Asia Oriental (sobre todo a China) no son suficientes para compensar todo lo que se está importando de allí, de modo que de no revertirse pronto esta tendencia sólo dos caminos quedarán para compensar dichos déficits: o bien aumentar el endeudamiento con aquella región o bien exacerbar el extractivismo y aumentar así la producción de bienes primarios - lo que podría derrumbar sus precios por la suba de la oferta y generar aún mayor ahogo productivo-, con lo cual, en cualquiera de los dos casos es posible que los déficits comerciales no se compensen, sino que agraven el patrón económico que los generó al acentuar la primarización económica, multiplicar los proyectos extractivos, acentuar de los conflictos socioambientales, etc. Es decir, existe el peligro real de edificar mayor destrucción y saqueo en los lugares donde se prometió la llegada del progreso. Por lo cual, el modelo económico de América Latina del «siglo XXI» puede encontrarse presa de un «esquema siglo XIX», en el cual el crecimiento no sea autocentrado y en base a un proceso de valor agregado cada vez mayor, sino de complementariedad y de adaptación subordinada frente a las nuevas potencias mundiales asiáticas, sin romper las inmensas asimetrías que ya caracterizan el vínculo entre ambas regiones.

El proceso que tiene al litio como protagonista no puede comprenderse por fuera de las estructuras de poder económico y político a nivel mundial, regional o local. En el escenario de la geopolítica internacional, la disputa global por los minerales estratégicos será central para direccionar los movimientos de los productores, consumidores, proveedores e inversores. Así, la dinámica no afecta sólo al ámbito de la esfera comercial, sino al tejido productivo, extractivo, las poblaciones, los patrones tecnológicos y al medioambiente, 
siendo en todos los casos centrales el dominio y gestión de los recursos naturales. Sin una mirada estratégica que sea capaz de repensar el rol de la soberanía en una visión de largo plazo, que les permita a los países latinoamericanos considerarse de manera integrada para dejar atrás los problemas de la dependencia, los problemas se agravarán. En este sentido, replantear el tipo de vínculo que se debe construir con el ascendiente asiático no debe descuidarse. Por lo que no dejará de ser vital para los países que poseen el recurso trazar algún tipo de estrategia, ya sea individual o conjunta, en el proceso de la transición económica y energética que ya ha comenzando. Por ejemplo, establecer economías de escala entre los países para fabricar, en forma conjunta, la batería de litio y asegurarse la producción local de esto, por lo menos en el subcontinente, protegiendo así a esta incipiente industria. También puede alentarse la cooperación internacional en investigación, ciencia y desarrollo, a través de las universidades y centros científicos. Aunque, por ahora, ninguna de estas chances ha avanzado y son sólo posibilidades a desarrollar hacia el futuro.

Para los países del denominado «triángulo del litio» los desafíos no son menores. Por un lado, por la gran cantidad de problemas virtuales que señalamos arriba. Pero por otro, porque les cabe también llevar a cabo, en el mejor de los casos, un proceso de intervención y coordinación que logre superar obstáculos en los que fácilmente se caerá si no se actúa de forma correcta. Uno de estos obstáculos es la sobreproducción, a la que no es muy difícil llegar en un mercado tan chico como el del litio y que tanta expectativa ha despertado. Así, en la actualidad todavía hay muchos salares que no han entrado en proceso de producción, pero que sí han comenzado sus etapas previas. Por lo cual, la cantidad ofrecida subirá y si lo hace más rápido que la demanda los precios caerán perdiendo la atractiva rentabilidad que la provocó. En la Argentina, por ejemplo, a fines de 2014 entró en proceso de producción plena el Salar de Olaroz con 16000 toneladas anuales de carbonato de litio. Próximamente también lo hará Bolivia con el Salar de Uyuni, el más importante del mundo. De no mediar formas de actuar que regulen la oferta sucederá lo mismo que en 1997, cuando ingresó la empresa chilena SQM y deprimió los precios un 40\%. El difícil dilema parece ser, entonces, el de actuar por delante de los acontecimientos, previendo problemas sin resignar el futuro sudamericano a ser un mero proveedor de materias primas, pero - a su vez - que las intervenciones se den con la suficiente flexibilidad para no aplastar los procesos que todavía no se han consolidado, implicando la necesidad de hacer adaptaciones frente a los imprevistos. De no actuar de esta forma, se repetirán los errores del pasado. Errores que, tristemente, sabemos las consecuencias que tuvieron para nuestros pueblos.

\section{REFERENCIAS}

¿Cuáles son los impactos ambientales y la salud de la explotación de litio? (2012, agosto 10). No a la mina. Recuperado de http://www.noalamina.org/mineria-preguntas-frecuentes/mineria-general/ icuales-son-los-impactos-ambientales-y-la-salud-de-la-explotacion-de-litio

Acosta, A. (2011). Extractivismo y Neoextractivismo: dos caras de la misma maldición. En AA.VV. Más allá del desarrollo (pp.83-118). Quito, Ecuador: Editorial Abya Yala.

Bruckman, M. (2012). Recursos naturales y la geopolítica de la integración Sudamericana. Lima, Perú: Fondo Editorial Mariatégui. 
Casalis, A. y Trinelli, A. (2013). El desarrollo territorial en la Argentina. Oportunidades y desafíos de la explotación de los recursos mineros (2002-2012). Revista Estado y Políticas Públicas, (1), 97-114.

CEDHA (2012). El nuevo horizonte minero. Dimensiones sociales, económicas y ambientales. Córdoba, Argentina: CEDHA.

CEPAL (2010). La inversión extranjera directa en América Latina y el Caribe. Santiago de Chile, Chile: Cepal.

COCHILCO (2009). Antecedentes para una política pública en minerales estratégicos: Litio. Santiago de Chile, Chile: Dirección de Estudios y políticas públicas.

Féliz, M. (2011). Neoliberalismos, neodesarrollismos y proyectos contrahegemónicos en Suramérica. Astrolabio, (7), 238-265.

FMC (2011). Lithium Market Review. Santiago de Chile, Chile: FMC.

Gacetilla del área minera (2011). Universidad Nacional de Jujuy, Facultad de Ingeniería, (163), s/p.

Gudynas, E. (2011). Debates sobre el desarrollo y sus alternativas en América Latina: Una breve guía heterodoxa. En AA.VV., Más allá del desarrollo (pp.21-53). Quito, Ecuador: Editorial Abya Yala. Ministerio de Economía de Argentina (2011). Complejo minero: litio. Buenos Aires, Argentina.

Puente, F. y Wahren, J. (2014). Dinámicas territoriales frente a la explotación del litio en Argentina. Ponencia presentada en las II Jornadas de Estudios de América Latina y el Caribe. Realizado por el IEALC. Buenos Aires, Aergentina.

Riva Palacio, L. E. (2012). Del triángulo del litio y el desarrollo sustentable. Una crítica del debate sobre la explotación en Sudamérica en el marco del desarrollo capitalista. México DF, México: Observatorio Latinoamericano de Geopolítica, UNAM.

Rodríguez, G. (2011). La participación de América Latina en el G20. En AA.VV., El G20, América Latina y el futuro de la integración regional (s/p). Washington, Estados Unidos: Fundación Heinrich Böll Stiftung.

Rosales, O. y Kuwayama, M. (2012). China y América Latina y el Caribe. Hacia una relación económica $y$ comercial estratégica. Santiago de Chile, Chile: Cepal.

Rubiolo, M. y Baroni, P. (2014). El sudeste de Asia en sus vinculaciones Sur-Sur de la Argentina: ¿Es la diversificación suficiente? Revista de Estudos \& Pesquisas sobre as Américas, 8(2), 215-233.

Sevares, J. y Krzemien, J. (2012). El litio en la Argentina: oportunidades y desafíos de un recurso estratégico. Realidad Económica, (272), s/p.

Slipak, A. (2014a). América Latina y China: ¿cooperación Sur-Sur o 'Consenso de Beijing’?. Nueva Sociedad, (250), 102-113.

Slipak, A. (2014b). La expansión de China en América Latina: incidencia en los vínculos comerciales argentino-brasileros, Congreso de Economía Política Internacional, Universidad Nacional de Moreno (UNM). Buenos Aires, Argentina.

Sukup, V. (2000). Asia frente al siglo XXI. ¿De los 'milagros' a las 'debacles'? Buenos Aires, Argentina: Corregidor.

Svampa, M. (2013). Consenso de los commodities y lenguajes de valoración en América Latina. Nueva Sociedad, (244), s/p.

Svampa, M. y Slipak, A. (2015). China y la nueva dependencia de América Latina: Del Consenso de los Commodities al Consenso de Beijing. VIII Jornadas de Economía Crítica. Río Cuarto. 